This is an electronic reprint of the original article. This reprint may differ from the original in pagination and typographic detail.

Author(s): Arjoranta, Juho; Heikkilä, Tero

Title: Intrinsic spin-orbit interaction in diffusive normal wire Josephson weak links:

Supercurrent and density of states

Year: $\quad 2016$

Version:

Please cite the original version:

Arjoranta, J., \& Heikkilä, T. (2016). Intrinsic spin-orbit interaction in diffusive normal wire Josephson weak links: Supercurrent and density of states. Physical Review B, 93(2), Article 024522. https://doi.org/10.1103/PhysRevB.93.024522

All material supplied via JYX is protected by copyright and other intellectual property rights, and duplication or sale of all or part of any of the repository collections is not permitted, except that material may be duplicated by you for your research use or educational purposes in electronic or print form. You must obtain permission for any other use. Electronic or print copies may not be offered, whether for sale or otherwise to anyone who is not an authorised user. 


\title{
Intrinsic spin-orbit interaction in diffusive normal wire Josephson weak links: Supercurrent and density of states
}

\author{
Juho Arjoranta and Tero T. Heikkilä \\ University of Jyvaskyla, Department of Physics and Nanoscience Center, P.O. Box 35, 40014 University of Jyväskylä, Finland
}

(Received 8 July 2015; revised manuscript received 8 December 2015; published 27 January 2016)

\begin{abstract}
We study the effect of the intrinsic (Rashba or Dresselhaus) spin-orbit interaction in superconductor-nanowiresuperconductor (SNS) weak links in the presence of a spin-splitting field that can result either from an intrinsic exchange field or the Zeeman effect of an applied field. We solve the full nonlinear Usadel equations numerically [The code used for calculating the results in this paper is available in https://github.com/wompo/Usadel-fornanowires] and analyze the resulting supercurrent through the weak link and the behavior of the density of states in the center of the wire. We point out how the presence of the spin-orbit interaction gives rise to a long-range spin triplet supercurrent, which remains finite even in the limit of very large exchange fields. In particular, we show how rotating the field leads to a sequence of transitions between the 0 and $\pi$ states as a function of the angle between the exchange field and the spin-orbit field. Simultaneously, the triplet pairing leads to a zero-energy peak in the density of states. We proceed by solving the linearized Usadel equations, showing the correspondence to the solutions of the full equations and detail the emergence of the long-range supercurrent components. Our studies are relevant for ongoing investigations of supercurrent in semiconductor nanowires in the limit of several channels and in the presence of disorder.
\end{abstract}

DOI: 10.1103/PhysRevB.93.024522

\section{INTRODUCTION}

The antagonistic nature of conventional singlet superconductivity and magnetism has been clearly illustrated in experiments studying supercurrents flowing through ferromagnetic weak links $[1,2]$. There, the spin-splitting (exchange) field $h$ suppresses the supercurrent within a typically short magnetic length scale $\xi_{m}=\sqrt{\hbar D / h}$, where $D$ is the diffusion constant of the wire. As suggested in Ref. [3], this suppression can be lifted by converting part of the singlet supercurrent into a triplet with a finite projection of the magnetic moment of Cooper pairs by utilizing an inhomogeneous magnetization at the interface between the ferromagnet and the superconductor. This component couples electrons with spins from the same band, and therefore it is not sensitive to the spin-splitting field. This suggestion was experimentally demonstrated in a number of works [4,5] utilizing a series of different types of magnetic layers that are noncollinear with respect to each other.

Besides using magnetic materials, the spin-splitting field can be realized via the Zeeman effect of an applied magnetic field [6,7]. Similar physics as in the superconductorferromagnet-superconductor case can be envisaged as long as the orbital effect of the magnetic field is weak enough $[8,9]$ and does not limit the supercurrent. Such a situation takes place especially in narrow nanowires, where the spinsplitting field in combination with the Rashba-type spin-orbit (SO) interaction has been used in an effort to take these wires to the limit of topological superconductivity [10-12] for the detection of Majorana-type excitations at the edges of the wires. Most of such experiments are nevertheless in the topologically trivial limit. It is hence of interest to study the physics of such nanowires in the presence of the combination of the spin-orbit and spin-splitting fields. This is the aim of the present work. In particular, we study the supercurrent behavior in systems schematically presented in Fig. 1. Contrary to many recent theory works on the effects of spin-orbit coupling on proximity superconductivity discussing the fully ballistic regime [13-15], we assume the wires to be diffusive. Strictly speaking this limit requires that all wire dimensions are smaller than the elastic mean free path. Typical epitaxial nanowires have mean free paths comparable to the wire thickness and much less than the wire length [16-23]. Even in this limit the diffusive-limit theory is likely to capture the essential physics much better than the fully ballistic limit. In the diffusive limit it is generally possible to obtain a fully quantitative fit with between theory and experiments $[24,25]$, which is why also the quantitative details of the theory are relevant. On the other hand, the quasiclassical theory we employ corresponds to setting the Fermi wavelength $\lambda_{F} \rightarrow 0$. Therefore it cannot capture effects related to, for example, weak antilocalization, possibly relevant in these wires $[23,26,27]$. Alternative derivations of the quasiclassical theory on the fixed few-channel limit [28] cannot be directly connected to the many-channel limit considered here.

This paper extends on the work of Bergeret and Tokatly $[29,30]$, who introduced the mechanism of including the intrinsic (Rashba or Dresselhaus) spin-orbit interaction as a spindependent vector potential into the Usadel equation describing inhomogeneous superconductivity in the diffusive limit. They also pointed out how for certain relative orientations of the wire, spin-orbit fields, and the exchange field, the combination of the latter two may produce a triplet supercurrent that survives even at large exchange fields. In particular, they showed that the wire with a homogeneous exchange field and intrinsic spin-orbit interaction is gauge equivalent to a ferromagnet with inhomogeneous magnetization (see also Ref. [31]). Here we study this mechanism quantitatively (Sec. IV). In particular, we show the dependence of the supercurrent vs exchange field for varying magnitudes of the Rashba field. We also demonstrate in detail how the triplet supercurrent depends on the direction of the magnetic field applied in the plane of the wire. For strong spin-orbit coupling, we predict 


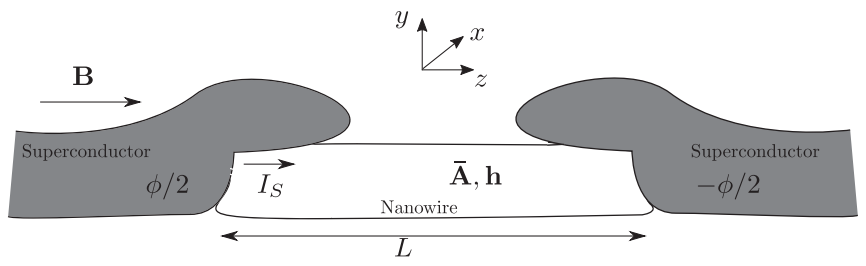

FIG. 1. SNS junction studied in this work. A diffusive nanowire of length $L$ connects two bulky superconductors. The nanowire is characterized by the spin-orbit field $\overline{\mathbf{A}}$ and the system exhibits an exchange field $\mathbf{h}$, either due to an applied magnetic field $\vec{B}$ or by proximity to magnetic material (not in the picture). We assume that both $\overline{\mathbf{A}}$ and $\mathbf{h}$ are in the $x z$ plane and that $\mathbf{h}$ is at an angle $\theta$ compared to the $(z)$ direction of the wire.

that changing the direction of the field drives the junction through a sequence of $0-\pi$ transitions.

Besides supercurrent, we also study the local density of states in the junction in Sec. V. This is also the typical observable in the studies of Majorana physics. We find that spin-orbit interaction induces a zero-energy peak for a range of exchange fields. This peak originates from the induced long-range triplet amplitude of the superconducting pairing, and it is quite sensitive to the precise direction of the field and the amplitude of the SO coupling.

\section{USADEL EQUATION WITH SPIN-ORBIT COUPLING}

We implement the spin-orbit interaction into the Usadel equation describing the quasiclassical Nambu-spin retarded Green's function $\hat{G}^{R}$ in the diffusive limit [29,32,33] (here and below, $e=\hbar=k_{B}=1$ except when we discuss particular values of the observables):

$D \hat{\nabla} \cdot\left(\hat{G}^{R} \hat{\nabla} \hat{G}^{R}\right)=\left[-i \varepsilon \hat{\tau}_{3}-i \mathbf{h} \cdot \bar{\sigma}+\hat{\Delta}+\hat{\Sigma}_{\mathrm{sf}}+\hat{\Sigma}_{\mathrm{in}}, \hat{G}^{R}\right]$.

Here $D$ is the diffusion constant inside the nanowire, $\varepsilon$ is the energy, $\bar{\sigma}$ is a vector of Pauli matrices in spin space, $\mathbf{h}=$ $\left(h_{x}, h_{y} \hat{\tau}_{3}, h_{z}\right)$, and $\hat{\nabla} \hat{G}^{R}=\nabla_{\mathbf{R}} \hat{G}^{R}-i\left[\overline{\mathbf{A}} \hat{\tau}_{3}, \hat{G}^{R}\right]$ is the gauge invariant gradient with $\overline{\mathbf{A}}$ describing the SO coupling. The latter is specified below in more detail [34].

Inside the normal metal, the superconducting pair potential $\hat{\Delta}$ is zero and we assume that the term $\hat{\Sigma}_{\text {in }}$ describing the inelastic scattering is negligible. In practice, the junction contains also regular spin-flip scattering and scattering due to isotropic spin-orbit coupling [35], characterized by the self-energy $\hat{\Sigma}_{\text {sf }}$. We assume spin relaxation to be dominated by the intrinsic SO coupling and neglect these other terms in the following. Thus inside the normal metal we have

$$
D \hat{\nabla} \cdot\left(\hat{G}^{R} \hat{\nabla} \hat{G}^{R}\right)=\left[-i \varepsilon^{+} \hat{\tau}_{3}-i \mathbf{h} \cdot \bar{\sigma}, \hat{G}^{R}\right],
$$

where $\varepsilon^{+}=\varepsilon+i \eta$, and $\eta \rightarrow 0^{+}$is a small term specifying the location of the poles of the retarded Green's function.

Introducing a dimensionless position coordinate $z^{\prime}=z / L$ and defining a Thouless energy $E_{T}=D / L^{2}$, where $L$ is the length of the normal metal wire, we can work in energy units of $\varepsilon=E / E_{T}$ and $\tilde{\mathbf{h}}=\mathbf{h} / E_{T}$ and use the scaled vector potential $\overline{\mathbf{A}}_{s}=\overline{\mathbf{A}} L$. Moreover, below we assume the Zeeman field to lie in the substrate plane, and therefore $h_{y}=0$. Equations (1) and
(2) have to be supplemented with the normalization condition $\left(\hat{G}^{R}\right)^{2}=\hat{1}$. In the numerical solutions, we implement this by using the Riccati parametrization [36] (see Appendix A) for the retarded Green's function. In that parametrization, the Nambuspace Green's function is specified in terms of two parameters, $\gamma$ and $\tilde{\gamma}$. In general, both of these parameters are $2 \times 2$ matrices in spin space. The Usadel equation written for $\gamma$ reads

$$
\begin{aligned}
\partial_{z^{\prime}}^{2} \gamma & -2\left(\partial_{z^{\prime}} \gamma\right) \tilde{\gamma} N\left(\partial_{z^{\prime}} \gamma\right) \\
= & -2 i \epsilon \gamma-i \tilde{\mathbf{h}} \cdot[\gamma, \bar{\sigma}]+\left[\overline{\mathbf{A}}_{s}^{2}, \gamma\right]+2\left\{\overline{\mathbf{A}}_{s}, \gamma\right\} \tilde{N}\left(\overline{\mathbf{A}}_{s}-\tilde{\gamma} \overline{\mathbf{A}}_{s} \gamma\right) \\
& +2 i\left[\left(\partial_{z^{\prime}} \gamma\right) \tilde{N}\left(\overline{\mathbf{A}}_{s}-\tilde{\gamma} \overline{\mathbf{A}}_{s} \gamma\right)+\left(\overline{\mathbf{A}}_{s}-\gamma \overline{\mathbf{A}}_{s} \tilde{\gamma}\right) N\left(\partial_{z^{\prime}} \gamma\right)\right] . \quad(3)
\end{aligned}
$$

For equilibrium observables, we replace $-i \epsilon$ by the Matsubara frequencies $\omega_{n}=\pi T(2 n+1)$ [37]. The equation for $\tilde{\gamma}$ is obtained by substituting $\gamma \leftrightarrow \tilde{\gamma}, N \leftrightarrow \tilde{N}$ and by taking a complex conjugate of the scalars.

\section{A. Spin-orbit field}

A generic spin-orbit coupling emerging in systems with broken inversion symmetry is of the form $\bar{A}_{i}=\alpha_{i}^{j} \bar{\sigma}_{j}$. For a one-dimensional wire the symmetry is broken by the geometry in both directions perpendicular to the wire, and therefore the components $\alpha_{i}^{j}$ can be considered independent of each other. To describe a one-dimensional wire in the $z$ direction placed on a substrate (see Fig. 1) spanning the $x z$ plane, we describe the generic spin-orbit field as

$$
\overline{\mathbf{A}}=\left(\alpha_{1}^{1} \bar{\sigma}_{1}+\alpha_{1}^{3} \bar{\sigma}_{3}, \quad 0, \quad \alpha_{3}^{1} \bar{\sigma}_{1}+\alpha_{3}^{3} \bar{\sigma}_{3}\right) .
$$

For a thin wire there are gradients only in the $z$ direction, and therefore the only terms coupling linearly to the gradient are related to $\alpha_{3}$. The other terms can be nonvanishing and they contribute in general to the Dyakonov-Perel spin relaxation $[29,30]$, but as they do not lead to other interesting physics, we neglect them in our numerical results. After neglecting the orbital effect of the field, the properties of the junction depend only on the relative direction between $\mathbf{h}$ and $\vec{A}_{z}$ in $\vec{A}_{z}=\vec{A}_{z} \cdot \vec{\sigma}$. As we vary the direction of $\mathbf{h}$, we fix $\bar{A}_{z} \propto \sigma_{1}$, i.e., $\alpha_{3}^{3}=0$. We vary the remaining term $\alpha=\alpha_{3}^{1}$ to observe the physics due to the intrinsic spin-orbit coupling. Note that the term $\alpha \sigma_{1} \hat{u}_{z}$ gives rise to the linear energy term of the form $e \alpha \sigma_{1} p_{z} / m$. Our term $\alpha$ is related to the (Rashba) terms of size $\tilde{\alpha}$ considered in the literature on Majorana fermions (e.g., [38]) by

$$
\alpha=\frac{m \tilde{\alpha}}{\hbar e},
$$

where $m$ is the effective mass of the electrons inside the nanowire. Our $\alpha$ hence has a dimension of $\hbar /(e L)$, where $L$ is some length scale. In the numerics, the important dimensionless parameter is $e \alpha L / \hbar$, where $L$ is chosen to be the distance between the two nanowire-superconductor contacts. For example, with the parameters of InSb discussed in Ref. [38] ( $m=0.015 m_{e}$ and $\tilde{\alpha}=0.2 \mathrm{eV} \AA$, where $m_{e}$ is the electron mass), we get $e \alpha / \hbar \approx 4 \times 10^{6} 1 / \mathrm{m}$. These estimates are consistent with recent experiments [23], which obtain even a somewhat larger value of $\tilde{\alpha}$. For typical wires of length $L \sim$ $1 \mu \mathrm{m}$, the value of the dimensionless parameter $e \alpha \hbar L$ can hence be of the order of or larger than unity. On the other hand, in order to be able to neglect gradients in transverse directions 
in Eq. (3), we assume that the wires are narrow compared to the spin-orbit length, i.e., wire thickness $d$ satisfies $d \ll \hbar / e \alpha$.

\section{B. Boundary conditions}

For the boundary conditions to the Green's functions, we assume clean NS interfaces. This assumption means that the parameters $\gamma$ and $\tilde{\gamma}$ are continuous across the interface and coincide with bulk BCS superconductor values [33,39],

$$
\begin{aligned}
\gamma_{\mathrm{BCS}} & =\frac{i|\Delta| e^{i \varphi}}{\varepsilon+i \sqrt{|\Delta|^{2}-\left(\varepsilon+i 0^{+}\right)^{2}}} \bar{\sigma}_{2}, \\
\tilde{\gamma}_{\mathrm{BCS}} & =\frac{i|\Delta| e^{-i \varphi}}{\varepsilon+i \sqrt{|\Delta|^{2}-\left(\varepsilon+i 0^{+}\right)^{2}}} \bar{\sigma}_{2},
\end{aligned}
$$

in the real-time description (for Matsubara frequencies, replace $\epsilon$ by $\left.i \omega_{n}\right)$. The exact form of the boundary conditions depends on the chosen form of the Nambu vector.

In the numerics, we express all the lengths in terms of the length $L$ of the wire. In this case, the natural energy scale is given by the Thouless energy $E_{T}=\hbar D / L^{2}$. We mostly concentrate on the limit of long wires, where $L \gg$ $\xi_{0}=\sqrt{\hbar D / \Delta}$. This also means that $\Delta / E_{T}=L^{2} / \xi_{0}^{2} \gg 1$.

\section{Supercurrent}

The supercurrent through the junction is characterized by the spectral current density [40]

$$
j_{s}=\frac{L}{4} \operatorname{Tr}\left[\left(\hat{G}^{R} \hat{\nabla} \hat{G}^{R}-\hat{G}^{A} \hat{\nabla} \hat{G}^{A}\right) \hat{\tau}_{3}\right] .
$$

In the Riccati parametrization, the spectral current density can be written as

$$
\begin{aligned}
j_{s}= & \frac{L}{2}\left\{\operatorname { T r } \left[N\left(\gamma \tilde{\gamma}^{\prime}-\gamma^{\prime} \tilde{\gamma}\right) N-\tilde{N}\left(\tilde{\gamma} \gamma^{\prime}-\tilde{\gamma}^{\prime} \gamma\right) \tilde{N}\right.\right. \\
& +i(N\{\overline{\mathbf{A}}, \gamma\} \tilde{\gamma} N+N \gamma\{\overline{\mathbf{A}}, \tilde{\gamma}\} N \\
& +\tilde{N}\{\overline{\mathbf{A}}, \tilde{\gamma}\} \gamma \tilde{N}+\tilde{N} \tilde{\gamma}\{\overline{\mathbf{A}}, \gamma\} \tilde{N})]\},
\end{aligned}
$$

where $\gamma^{\prime}=\partial \gamma / \partial z$.

The supercurrent can be calculated as a weighted average of the spectral current density

$$
I_{s}=\frac{E_{T}}{2 e R_{N}} \int_{-\infty}^{\infty} d \varepsilon \operatorname{Re} j_{s}(\varepsilon) \tanh \left(\frac{\varepsilon}{2 T}\right),
$$

where $R_{N}=L /\left(A e^{2} D v_{F}\right)$ is the Drude resistance of the nanowire in the normal state. Here $L$ is the length, $A$ is the area, $D$ the diffusion constant, and $v_{F}$ the density of states at the Fermi level of the nanowire in its normal state. In the Matsubara technique, the integral can be calculated as a sum of the spectral current densities evaluated at the Matsubara frequencies $\omega_{n}$ as

$$
I_{s}=-\frac{E_{T}}{e R_{N}} 2 \pi T \sum_{n=0}^{\infty} \operatorname{Im} j_{s}\left(\omega_{n}\right) .
$$

In the numerics, we cut the Matsubara sum to the index after which the obtained supercurrent changes less than $1 \%$.

\section{Density of states}

The density of states (DOS) is given by [33]

$$
N(\varepsilon, \mathbf{R})=\frac{N_{F}}{2} \operatorname{Re}\left\{\operatorname{Tr}\left[\hat{G}^{R}(\varepsilon, \mathbf{R}) \hat{\tau}_{3}\right]\right\},
$$

where $N_{F}$ is the DOS in the absence of superconductivity. In the Riccati parametrization,

$$
N(\varepsilon, \mathbf{R})=\frac{N_{F}}{2} \operatorname{Re} \operatorname{Tr}\{N(1-\gamma \tilde{\gamma})+\tilde{N}(1-\tilde{\gamma} \gamma)\} .
$$

Besides supercurrent, the density of states is another way to characterize the excitation spectrum induced by the proximity effect. It can be accessed via a standard tunneling measurement. In Sec. V we show how a combination of the finite exchange field and the Rashba spin-orbit coupling gives rise to a zero-energy peak in the density of states. This is qualitatively similar to what one expects from the measurements in the Majorana wires [10-12], although the physics of this effect is quite different. Initial results for the density of states in Rashba wires were presented by us in [41]. Recently, similar types of results were discussed also in Refs. [42,43], but that work concentrated on superconductor/ferromagnet multilayers in the short junction limit, where the junction length is of the order of the superconducting coherence length $\xi_{0}=\sqrt{\hbar D / \Delta}$. In such multilayers, the emergence of the longrange triplet superconductivity requires either the presence of both Rashba- and Dresselhaus-type spin-orbit coupling or out-of-plane magnetic fields. Moreover, for short junctions the energy scales are primarily set by the superconducting gap $\Delta$ instead of the Thouless energy as here. Nevertheless, also there the triplet proximity effect leads to the presence of zero-energy density of states peaks and long-range supercurrent. However, the quantitative details of the results are quite different, and therefore as such not applicable for the nanowire setups.

\section{GENERATION OF THE LONG-RANGE TRIPLET COMPONENT}

Below we present numerical solutions of the supercurrent and density of states in a nanowire Josephson weak link exhibiting both intrinsic spin-orbit coupling and exchange field. To understand these results, let us first study what we expect to find in the limit of a weak proximity effect $[29,30]$, which allows us to linearize the Usadel equation.

Linearizing Eq. (3) yields

$$
\begin{aligned}
\partial_{z^{\prime}}^{2} \gamma= & 2 \omega_{n} \gamma-i \tilde{\mathbf{h}} \cdot[\gamma, \bar{\sigma}]+\left[\overline{\mathbf{A}}_{s}^{2}, \gamma\right] \\
& +2\left\{\overline{\mathbf{A}}_{s}, \gamma\right\} \overline{\mathbf{A}}_{s}+2 i\left\{\partial_{z^{\prime}} \gamma, \overline{\mathbf{A}}_{s}\right\} .
\end{aligned}
$$

Here we have discarded all the terms $\mathcal{O}\left(\gamma^{2}\right)$ and noticed that $N=1$ due to the normalization condition $\left(\hat{G}^{R}\right)^{2}=1$. We separate the different spin components as

$$
\gamma=\sum_{i=0}^{3} f_{i} \bar{\sigma}_{i}
$$

and choose a specific SO field $\overline{\mathbf{A}}_{s}=\alpha \bar{\sigma}_{1} \hat{u}_{z}$ and direction of the magnetic field, $\tilde{\mathbf{h}}=h[\sin (\theta), 0, \cos (\theta)]$. The resulting equations describe the interplay between the short-range and long-range pairing components. 
We can solve the resulting second-order boundary value problem by separating the short-range and long-range components $f_{i}$, and using the previous to find effective boundary conditions for the latter. The details are given in Appendix B. As a result, we get the spectral supercurrent describing the long-range component. In the case of a perpendicular field and $\overline{\mathbf{A}}_{s}(\theta=0)$, the result reduces to

$$
\operatorname{Im}\left(j_{s}\right)=-\frac{|\Delta|^{2}}{\left(\omega_{n}+\sqrt{\omega_{n}^{2}+|\Delta|^{2}}\right)^{2}} \frac{8 \sqrt{2} \alpha^{2} D \sqrt{\tilde{\omega}_{n}}}{h \sinh \left(\sqrt{2} \sqrt{\tilde{\omega}_{n}}\right)} \sin (\phi),
$$

where $\phi$ is the phase difference between the two superconductors, and $\tilde{\omega}_{n}=\left(\omega_{n}+2 D \alpha^{2}\right) / E_{T}$ is the (dimensionless) Matsubara frequency modified by the pair-breaking effect from the spin-orbit coupling $\left(\omega_{n} \mapsto-i \epsilon\right.$ in the real-time formulation with energy $\epsilon$ ). Note that the spin-orbit coupling $\alpha$ plays here a dual role: first, it induces the long-range triplet component of the (spectral) supercurrent, and second, it induces pair-breaking effects. If we would include the ordinary spin-flip or spin-orbit scattering effects, they would (in the lowest order) induce the corresponding terms into $\tilde{\omega}$.

The observable supercurrent at a given temperature $T$ is obtained by summing over the Matsubara frequencies or integrated over the real energies as in Eqs. (9) and (10). At low temperatures $k_{B} T \lesssim E_{T}$ we may transform the Matsubara sum into an integral and obtain in the long-junction limit $E_{T}, e^{2} \hbar \alpha^{2} D \ll \Delta$ (restoring $e$ and $\left.\hbar\right)$ :

$$
\begin{aligned}
I_{S}(T \approx 0)= & \frac{32 E_{T} e \alpha^{2} D}{h R_{N} \hbar} e^{-2 \alpha L e / \hbar}\left(1+\frac{2 \alpha L e}{\hbar}+\frac{2 \alpha^{2} L^{2} e^{2}}{\hbar^{2}}\right) \\
& \times \sin (\phi) .
\end{aligned}
$$

Strictly speaking this is valid only for $2 e \alpha L / \hbar \gtrsim 1$, but this approximation fits numerical results reasonably well also for low $\alpha$. On the other hand, at high temperatures $k_{B} T \gg E_{T}$, it is enough to include only the lowest Matsubara frequency and the result is

$$
\begin{aligned}
I_{S}\left(T \gg E_{T}\right)= & \frac{|\Delta|^{2}}{\left(\pi k_{B} T+\sqrt{\pi^{2} k_{B}^{2} T^{2}+|\Delta|^{2}}\right)^{2}} \frac{8 E_{T} e \alpha^{2} D}{h R_{N} \hbar} \\
& \times \sqrt{x} e^{-\sqrt{x}},
\end{aligned}
$$

where $x=2 \pi k_{B} T / E_{T}+2 \alpha L e / \hbar$.

We compare the analytical result to the full numerics in Fig. 4. For large $\alpha \gtrsim 6 \hbar /(e L)$, the full theory shows a second maximum in the supercurrent, absent in this analytical approximation (see Fig. 4).

For a nonzero $\theta \in] 0, \pi / 2$ [, both long-range components $f_{0}$ and $f_{3}$ become nonzero. Due to their coupling, the supercurrent obtains terms oscillating with $\alpha L e \sin (\theta) / \hbar$. The resulting oscillations vs $\theta$ for $\alpha L e \gtrsim 1$ signal transitions between 0 and $\pi$ states, which would be observed as cusps in the dependence of the critical current on the field direction. This is qualitatively described in Appendix B and further explored in Fig. 5.

\section{SUPERCURRENT}

The effects of the spin-orbit coupling show up only in the presence of a nonzero exchange field in the wire. This

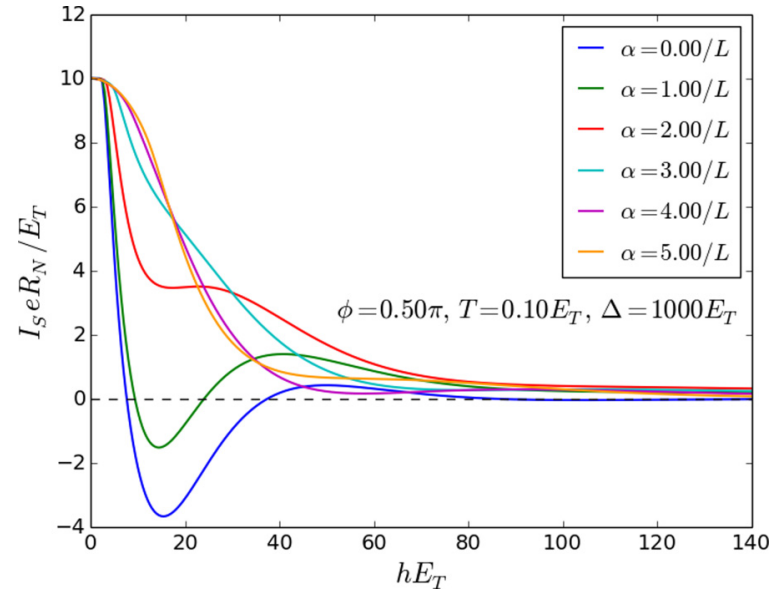

FIG. 2. Exchange field dependence of the supercurrent in a SNS junction for different intrinsic spin-orbit coupling strengths $\alpha$. A finite SO coupling strength yields a finite supercurrent through the junction even with large exchange fields.

exchange field can be established either in an intrinsically ferromagnetic wire, via the magnetic proximity effect in a wire in contact with a ferromagnetic insulator, or via the Zeeman field from an applied external magnetic field. In all these cases the (direct or stray) magnetic field generates also the orbital effect for the charge carriers. The relevance of this orbital effect depends on the aspect ratio of the wire [8,9]. Here we assume that the wire is thin enough so that we can disregard the orbital effect and concentrate only on the Zeeman field. Unless stated otherwise, the numerical results are obtained for a field in the direction of the wire (i.e., $\theta=0$ ).

The most straightforward experiment is to vary the magnitude of the exchange field. In Fig. 2 we plot the supercurrent $I_{S}$ at phase difference $\phi=\pi / 2$ (close to the critical current) at the temperature $T=0.1 E_{T}$ for a long junction (length $\left.\Delta / E_{T}=L^{2} / \xi_{0}^{2}=1000\right)$ as a function of the exchange field for varying magnitudes of the spin-orbit coupling $\alpha$. For low $\alpha \lesssim 1 / L$, the supercurrent exhibits damped oscillations as a function of the exchange field, as shown before, for example, in Refs. [44] and [1]. For $I_{S}<0$, the junction enters the $\pi$ state. With an increasing spin-orbit field, the oscillation of the supercurrent is suppressed and the junction no longer can be found in the $\pi$ state. (Note that nonquasiclassical corrections to our theory can lead to the presence of a $\phi$ state $[45,46]$ with $\phi \neq 0, \pi$ due to the spin-orbit coupling.) In the absence of spin-orbit coupling, $\alpha=0$, the supercurrent dies out for large exchange fields. However, a finite $\alpha$ yields also a finite supercurrent even at rather large exchange fields.

For an intermediate value of $\alpha=2 / L$, the supercurrent vs exchange field exhibits a minimum at $h \approx 10 \ldots 20 E_{T}$. This minimum persists to rather high temperatures, as shown in Fig. 3.

We can compare our results quantitatively to those obtained from the linearized Usadel equation at large values of the exchange field, where the major contribution to the supercurrent comes from the long-range components. In Fig. 4 we plot the supercurrent for a few values of the exchange field as a function of $\alpha$. The solid lines show the results from the exact numerics, whereas the dashed lines come from the Matsubara 


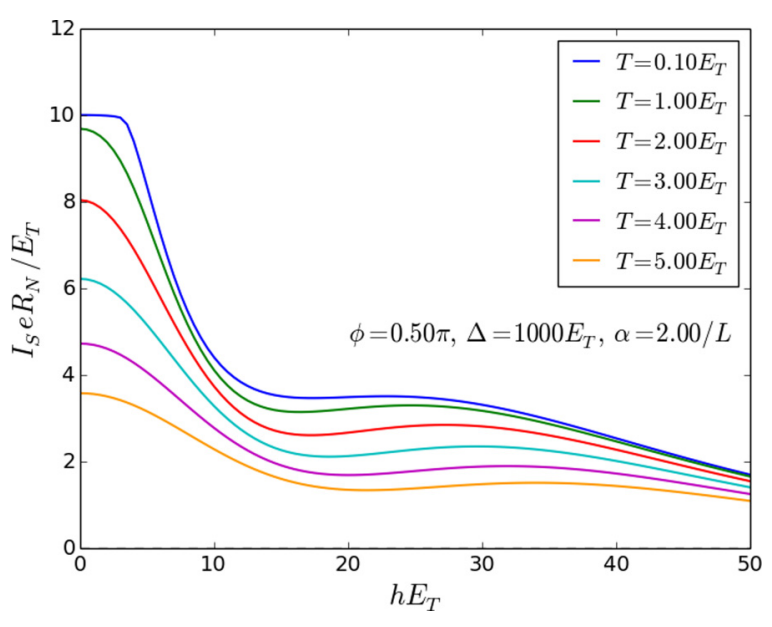

FIG. 3. Supercurrent through the SNS junction as a function of the exchange field at different temperatures.

sum of Eq. (15) [quite close to Eq. (16)]. We find that both approaches yield a supercurrent that is nonmonotonous with respect to the value of the spin-orbit field. Equations (15) and (16) capture the first oscillation rather well, but the amplitude of the exact supercurrent is somewhat larger than that obtained from the analytics.

Tokatly and Bergeret [29,30] showed that in order to get a long-range triplet supercurrent, the term describing the exchange field should not commute with the vector potential. We check this by investigating the dependence of the supercurrent on the angle $\theta$ of an in-plane exchange field with respect to the wire direction (see inset of Fig. 5). For $\theta=0$ the exchange field is in the $z$ direction and therefore produces the term proportional to $\bar{\sigma}_{3}$, whereas we describe the (Rashbatype) spin-orbit term proportional to $\bar{\sigma}_{1}$. For $\theta=\pi / 2$, both are described by terms proportional to $\bar{\sigma}_{1}$. The supercurrent $I_{S}(\theta)$ is plotted in Fig. 5. Indeed, for $\theta=\pi / 2, I_{S}(\theta)$ only contains singlet components of the pairing amplitude and therefore almost vanishes because of the large value of the

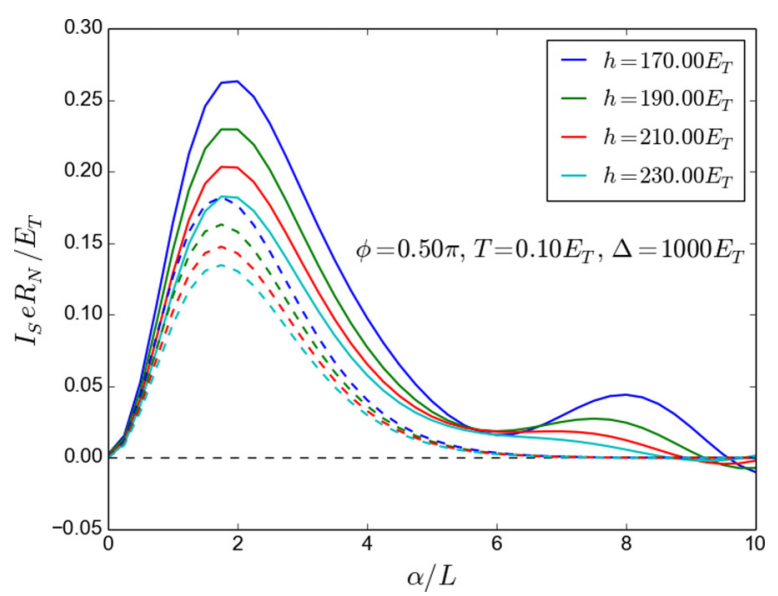

FIG. 4. With large exchange fields, the supercurrent oscillates as a function of the spin-orbit field strength, reaching a maximum at around $\alpha=2 / L$. The solid lines show the numerical results and the dashed lines are obtained by using Eq. (15) in Eq. (10).

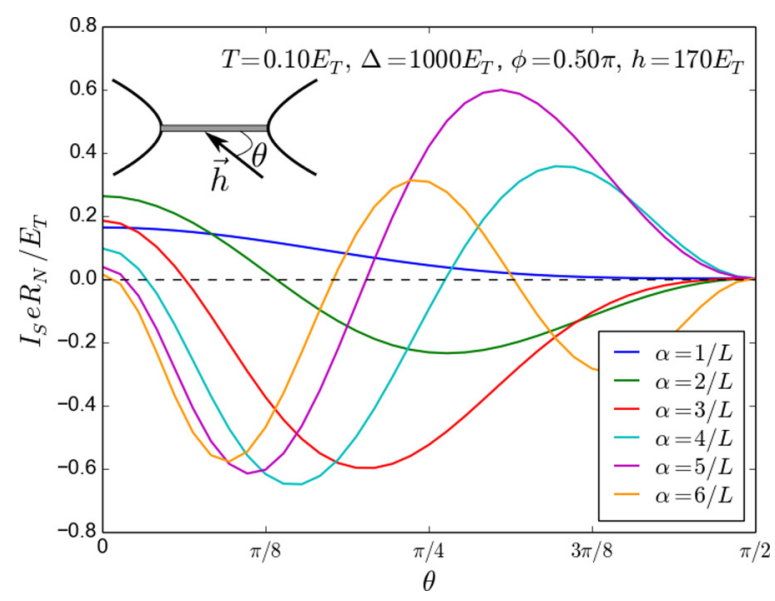

FIG. 5. Supercurrent as a function of the angle $\theta$ of the applied field, $\mathbf{h}=h\left[\cos (\theta) \hat{u}_{z}+\sin (\theta) \hat{u}_{x}\right], h=170 E_{T}$ and otherwise the same parameters as in Fig. 2. Rotating the exchange field makes the long-range component disappear when the spin-orbit field is parallel to the exchange field $(\theta=\pi / 2)$. In addition, applying a field at an intermediate angle results in an alternating sequence of $0-\pi$ transitions, their number depending on the precise value of $\alpha$.

chosen exchange field. In addition, as described in Appendix B, we find that for $\alpha \gtrsim 1 / L$, the junction shows a sequence of $0-\pi$ transitions, signalled by the sign change of supercurrent at $\phi=\pi / 2$. The magnitude of $\alpha$ dictates the positions of these transitions. For a large exchange field, these positions do not depend at all on the field and they only weakly depend on temperature. In experiments, $\alpha$ is not usually a variable quantity. However, the angle of the applied field can be varied straightforwardly. Therefore by studying the detailed angular dependence of the supercurrent, one is able to determine the magnitude and direction of the Rashba vector potential $\overline{\mathbf{A}}_{s}$. Note that the dependence of the supercurrent on $\theta$ resembles the behavior of supercurrent through a Bloch domain wall with a varying wave vector [47].

\section{DENSITY OF STATES}

Besides supercurrent, the proximity effect from the superconductor on the normal wire can be characterized via a tunneling measurement of the density of states. In the absence of the exchange field or the spin-orbit field, the density of states in the proximity wire exhibits a phase-dependent minigap [48] whose size can be approximatively described via $E_{g}(\phi) \approx 3.1 E_{T} \cos ^{2}(\phi / 2)$. In the absence of spin mixing (either via the intrinsic spin-orbit coupling considered here, spin-flip scattering, or the isotropic spin-orbit coupling), the exchange field simply shifts this minigap by $\sigma h$ for spin $\sigma= \pm$. For $h>E_{g}(\phi)$, we hence expect to see two regions with $N(E)=1 / 2$ in the spin-averaged density of states $N(E)$. The intrinsic spin-orbit coupling mixes the spins and leads to a closing of the minigaps for the spin-resolved density of states. As we show below, an intermediate magnitude of the intrinsic spin-orbit coupling also leads to a zero-energy peak in the density of states. The shape and height of this peak is very sensitive to the exact parameters of the system. We 


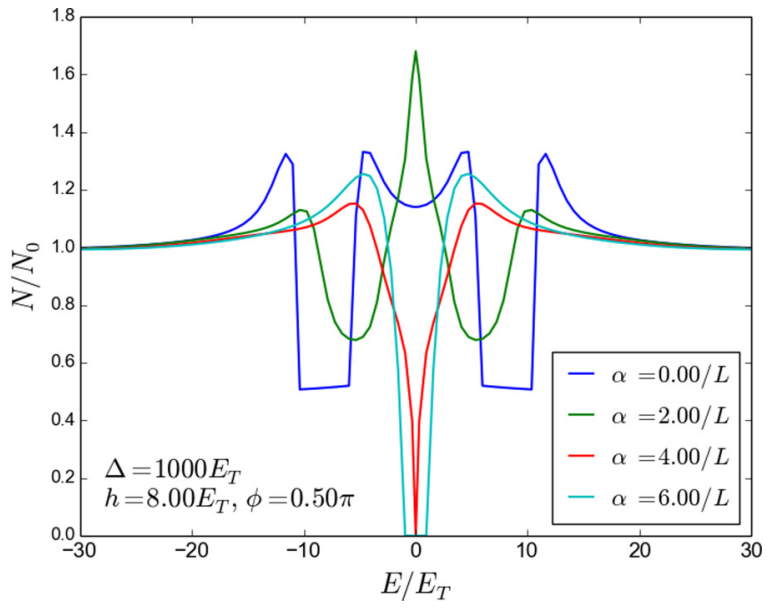

FIG. 6. Local density of states (DOS) in the center of the wire with $h=8 E_{T}$ and $\phi=0.5 \pi$ given with different spin-orbit fields. The DOS peaks at zero energy for $\alpha=2 / L$, while the peak converts to a zero-energy dip for larger $\alpha$.

illustrate these results in the following via a few examples; a more complete description can be found from Ref. [49].

The density of states is in general position dependent. To illustrate the proximity-induced effects in a symmetry point, we present the results calculated in the middle of the normal metal.

In Fig. 6 we show the DOS peaking at zero energy for $\alpha=2 / L$, while for larger $\alpha$ the peak converts to a zero-energy dip and again to an energy gap centered at around zero energy. As discussed in Refs. [42,50], the particular value for the zero-energy density of states results from the competition of the singlet proximity effect aiming to lower $N(0)$ and the long-range triplet proximity effect to increase it. This results from the different symmetry of the singlet vs triplet components. Namely, it is straightforward to show from the linearized equations, Eq. (B5), that for the singlet component $\tilde{f}_{2}(\epsilon=0)=f_{2}^{*}(\epsilon=0)$, whereas the triplet components satisfy $\tilde{f}_{j}(\epsilon=0)=-f_{j}^{*}(\epsilon=0), j \neq 2$. Expanding Eq. (12) to

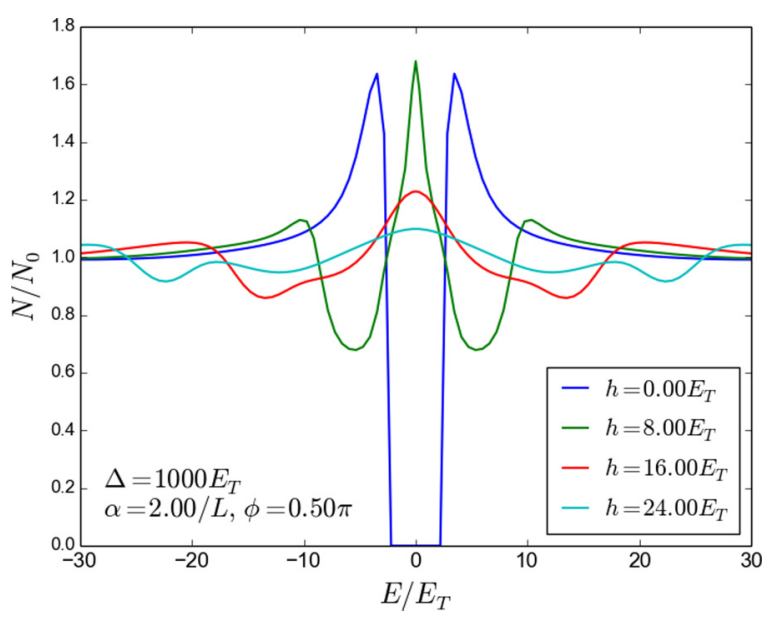

FIG. 7. Density of states with a constant $\alpha=2 / L$ and with different values of the exchange field $h$. The zero-energy peak forms only for $h \gtrsim 2 E_{g}(\phi)$.

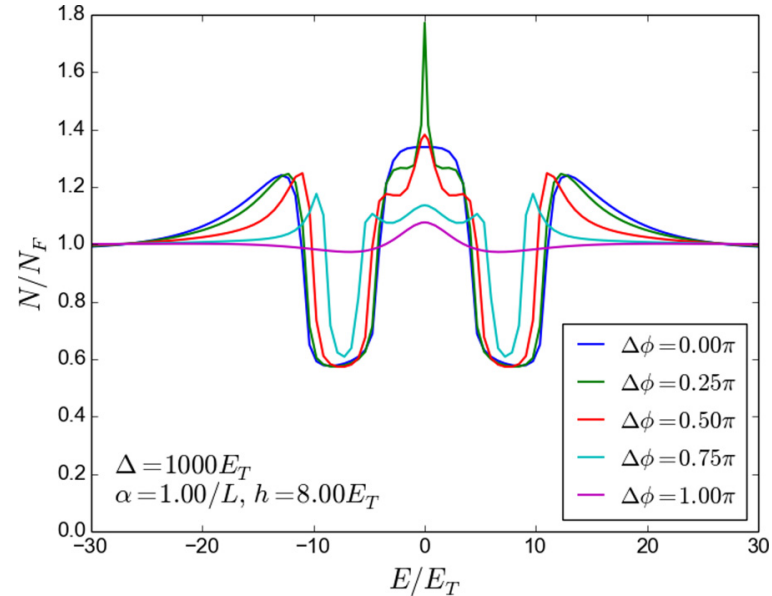

FIG. 8. Phase dependence of the DOS in the middle of the nanowire.

the lowest order in $\gamma$ then yields

$$
N(0) \approx N_{F}\left[1-2\left|f_{2}(0)\right|^{2}+2 \sum_{j \neq 2}\left|f_{j}(0)\right|^{2}\right] .
$$

For large $h$, the singlet component vanishes on a short distance $\sim \ell_{m}$ at the interface, whereas the long-range triplet components $f_{0,3}$ are much larger in the center of the wire. As a result, the latter yield $N(0)>1$. As shown in the figures, this may signal the presence of a zero-energy peak, but not necessarily. The values of $\alpha$ where the transition from a peak to a dip takes place are similar to those yielding a maximum in the long-range supercurrent, Fig. 4.

Although the origin is different from that expected for Majorana junctions [10-12], also for the diffusive nanowire Josephson junctions the zero-energy peak appears only at a large enough exchange field, in practice for $h \gtrsim 2 E_{g}(\phi)$ and for a restricted range of the values of $\alpha$. We plot the density of states at a constant $\alpha$ for a few different exchange fields in Fig. 7. The exact form of the density of states depends a lot on

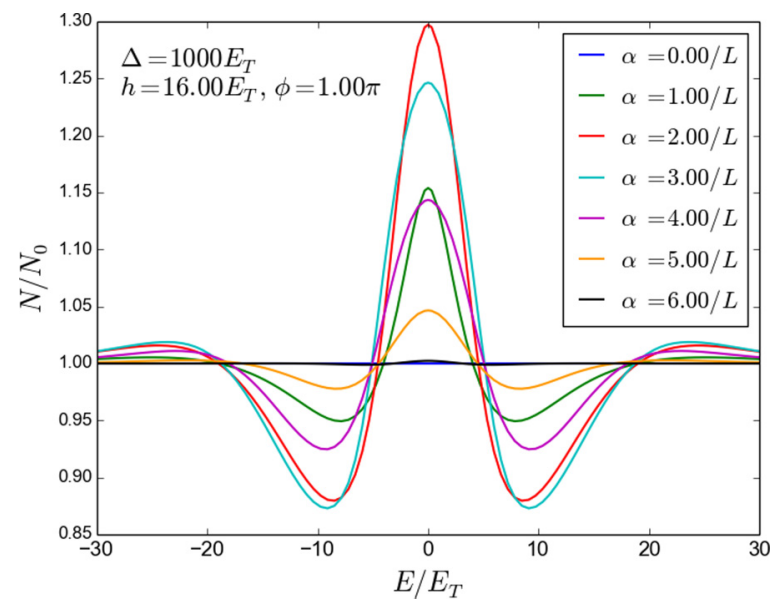

FIG. 9. Even with phase difference of $\phi=\pi$, the DOS peaks at zero energy for a finite SO coupling strength $\alpha$. 


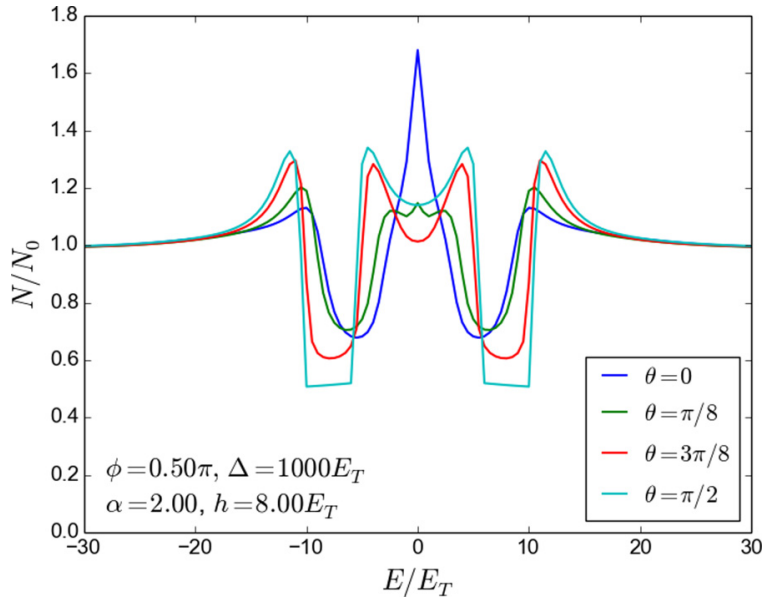

FIG. 10. Density of states for different directions of the exchange field, specified in terms of the angle $\theta$ (see inset of Fig. 5).

the value of the exchange fields, and the zero-bias peak decays at large values of $h$.

The density of states can also be controlled by applying a supercurrent through the junction so that the phase difference $\phi$ across the junction changes. Besides changing (and closing) the minigap for a vanishing exchange field, this changes the form of the density of states. The phase dependence is plotted in Fig. 8. Note that typically in long junctions $\left(\Delta \gg E_{T}\right.$ or $L \gg \xi_{0}=\sqrt{\hbar D / \Delta}$ ) the density of states for $\phi=\pi$ is almost featureless due to the destructive interference of the pair amplitudes emanating from the two superconductors. This is also modified by the spin-orbit coupling as shown in Fig. 9; also in this case a zero-energy peak forms.

Let us furthermore demonstrate the connection between the triplet proximity effect and the zero-energy peak [43,51-53]. Therefore, we study the density of states as a function of the angle $\theta$ of the exchange field between the $z$ direction of the wire and the $x$ direction, as in Fig. 5 for the supercurrent. This is shown in Fig. 10. For $\theta=\pi / 2$ we expect to get only the short-range proximity effect. In this case we indeed only find the exchange-field split minigaps, and the spin-orbit coupling makes almost no contribution to the form of the density of states.

\section{CONCLUSIONS}

In this work we have discussed the detailed effects of the Rashba- and Dresselhaus-type intrinsic spin-orbit interaction on the supercurrent carried through a diffusive nanowire. We solve the full Usadel equation to obtain the supercurrent of the junction and the local density of states. We reproduce the longrange triplet proximity effect predicted before in the linearized limit and show how the resulting supercurrent depends on the direction of the applied field. Besides the complicated direction dependence and the predicted zero-energy peaks and dips in the local density of states, our results pave the way of quantitatively analyzing the experiments carried out in the nanowire Josephson junctions. To reach, for example, the topological regime and confirm the Majorana character of the excitations in the nanowires, it is important that the experimentalists are able to characterize their junctions in detail. Our work gives a fixed point for such a characterization, in the (nontopological) many-channel diffusive limit. We hence expect this work to be relevant as an intermediate step for establishing the experimental constraints for using such junctions in topological quantum computing.

\section{ACKNOWLEDGMENTS}

We thank Pauli Virtanen, Sebastian Bergeret, and Timo Hyart for very useful discussions, and Francesco Giazotto, Attila Geresdi, and Charles Marcus for explaining the characteristics of InAs/InSb nanowires. This work was supported by the Academy of Finland through its Center of Excellence program under Project No. 284594, and by the European Research Council (Grant No. 240362-Heattronics).

\section{APPENDIX A: RICCATI PARAMETERIZATION}

The normalization condition $\left(\hat{G}^{R}\right)^{2}=1$ implies that the possible eigenvalues of $\hat{G}^{R}$ are \pm 1 . Therefore in spectral representation, $\hat{G}^{R}$ can be written in terms of the so-called Shelankov projectors as [54]

$$
\hat{G}^{R}=\hat{P}_{+}-\hat{P}_{-} \quad \text { with } \quad \hat{P}_{ \pm}=\frac{1}{2}\left(1 \pm \hat{G}^{R}\right) .
$$

The Shelankov projectors and the Green's function are convenient to parametrize in the Riccati parametrization [36],

$$
\hat{P}_{+}=\left(\begin{array}{cc}
N & N \gamma \\
\tilde{\gamma} N & \tilde{\gamma} N \gamma
\end{array}\right) \quad \text { and } \quad \hat{P}_{-}=\left(\begin{array}{cc}
\gamma \tilde{N} \tilde{\gamma} & -\gamma \tilde{N} \\
-\tilde{N} \tilde{\gamma} & \tilde{N}
\end{array}\right) \text {, }
$$

where $N=(1+\gamma \tilde{\gamma})^{-1}$ and $\tilde{N}=(1+\tilde{\gamma} \gamma)^{-1}$. Thus from Eq. (A1) the Green's function is

$$
\hat{G}^{R}=\left(\begin{array}{cc}
N & 0 \\
0 & \tilde{N}
\end{array}\right)\left(\begin{array}{cc}
1-\gamma \tilde{\gamma} & 2 \gamma \\
2 \tilde{\gamma} & -(1-\tilde{\gamma} \gamma)
\end{array}\right) .
$$

The projectors have the property

$$
\hat{\nabla} \hat{P}_{ \pm}= \pm \hat{P}_{+}[\hat{\nabla} U] \hat{P}_{-} \pm \hat{P}_{-}[\hat{\nabla} \tilde{U}] \hat{P}_{+},
$$

where

$$
U=\left(\begin{array}{ll}
0 & \gamma \\
0 & 0
\end{array}\right) \text { and } \tilde{U}=\left(\begin{array}{cc}
0 & 0 \\
\tilde{\gamma} & 0
\end{array}\right)
$$

In the spin-dependent case, $\gamma$ and $\tilde{\gamma}$ are $2 \times 2$-spin matrices, that is

$$
\gamma=\sum_{j=0}^{3} \gamma_{j} \bar{\sigma}_{j} \quad \text { and } \quad \tilde{\gamma}=\sum_{j=0}^{3} \tilde{\gamma}_{j} \bar{\sigma}_{j} .
$$

Using the above relations, we have derived the Usadel equation (3) and the spectral supercurrent, Eq. (8).

\section{APPENDIX B: LINEARIZED EQUATIONS}

Linearizing Eq. (3) yields

$$
\gamma^{\prime \prime}=2 \omega_{n} \gamma+i[\gamma, \bar{h}]+\left[\overline{\mathbf{A}}_{s}^{2}, \gamma\right]+2\left\{\overline{\mathbf{A}}_{s}, \gamma\right\} \overline{\mathbf{A}}_{s}+2 i\left\{\gamma^{\prime}, \overline{\mathbf{A}}_{s}\right\},
$$


where we use a shorthand notation $\partial_{z^{\prime}} \gamma=\gamma^{\prime}$. Writing

$$
\gamma=\sum_{i=0}^{3} f_{i} \bar{\sigma}_{i}
$$

assuming a general form for the SO coupling

$$
\overline{\mathbf{A}}=\left(\alpha_{1} \bar{\sigma}_{1}+\alpha_{2} \bar{\sigma}_{3}, \quad 0, \quad \alpha_{3} \bar{\sigma}_{1}+\alpha_{4} \bar{\sigma}_{3}\right),
$$

and choosing the exchange field in plane

$$
\bar{h}=h\left(\cos \theta \bar{\sigma}_{3}+\sin \theta \bar{\sigma}_{1}\right)
$$

we can separate the equations for the different spin components. They read

$$
\begin{aligned}
f_{0}^{\prime \prime}= & \left(2 \omega_{n}+4 \sum_{i=1}^{4} \alpha_{i}^{2}\right) f_{0}+4 i \alpha_{3} f_{1}^{\prime}+4 i \alpha_{4} f_{3}^{\prime}, \\
f_{1}^{\prime \prime}= & {\left[2 \omega_{n}+4\left(\alpha_{1}^{2}+\alpha_{3}^{2}\right)\right] f_{1}-2 h \cos \theta f_{2} } \\
& +4\left(\alpha_{1} \alpha_{2}+\alpha_{3} \alpha_{4}\right) f_{3}+4 i \alpha_{3} f_{0}^{\prime}, \\
f_{2}^{\prime \prime}= & 2 h \cos \theta f_{1}+2 \omega_{n} f_{2}-2 h \sin \theta f_{3}, \\
f_{3}^{\prime \prime}= & 4\left(\alpha_{1} \alpha_{2}+\alpha_{3} \alpha_{4}\right) f_{1}+2 h \sin \theta f_{2} \\
& +\left[2 \omega_{n}+4\left(\alpha_{2}^{2}+\alpha_{4}^{2}\right)\right] f_{3} .
\end{aligned}
$$

The equations for $\tilde{f}_{i}$ parametrizing $\tilde{\gamma}$ are obtained by substituting $f_{i} \leftrightarrow \tilde{f}_{i}, h \leftrightarrow-h$, and by taking a complex conjugate of the scalars.

The boundary conditions read

$$
f_{2}=c e^{i \phi / 2}, \quad \tilde{f}_{2}=c e^{-i \phi / 2}, \quad c=\frac{|\Delta|}{\omega_{n}+\sqrt{|\Delta|^{2}+\omega_{n}^{2}}},
$$

with $\phi$ having the opposite signs at $x=0$ and $x=L$. For $i=0,1,3$ the functions vanish at the boundaries, that is, $f_{i}(0)=f_{i}(L)=\tilde{f}_{i}(0)=\tilde{f}_{i}(L)=0$.

Linearizing the spectral current density [see Eq. (8)] yields

$$
\begin{aligned}
\operatorname{Im}\left(j_{s}\right)= & \frac{1}{2} \operatorname{Im}\left\{\operatorname { T r } \left[\left(\gamma \tilde{\gamma}^{\prime}-\gamma^{\prime} \tilde{\gamma}\right)-\left(\tilde{\gamma} \gamma^{\prime}-\tilde{\gamma}^{\prime} \gamma\right)\right.\right. \\
& +i(\{\overline{\mathbf{A}}, \gamma\} \tilde{\gamma}+\gamma\{\overline{\mathbf{A}}, \tilde{\gamma}\}+\{\overline{\mathbf{A}}, \tilde{\gamma}\} \gamma+\tilde{\gamma}\{\overline{\mathbf{A}}, \gamma\})]\} .
\end{aligned}
$$

Using the Pauli matrix expansion from Eq. (B2) and the general form for the SO coupling, Eq. (B3), we can simplify Eq. (B7),

$$
\begin{aligned}
\operatorname{Im}\left(j_{s}\right)= & \operatorname{Im}\left[\sum_{j=0}^{3}\left(f_{j} \tilde{f}_{j}^{\prime}-\tilde{f}_{j} f_{j}^{\prime}\right)\right. \\
& \left.+4 i\left[\alpha_{3}\left(f_{0} \tilde{f}_{1}+f_{1} \tilde{f}_{0}\right)+\alpha_{4}\left(f_{0} \tilde{f}_{3}+f_{3} \tilde{f}_{0}\right)\right]\right] .
\end{aligned}
$$

In the numerics we have chosen $\overline{\mathbf{A}}=\left(0,0,-\alpha \bar{\sigma}_{1}\right)$. On the other hand, the analytics becomes more straightforward by writing the $f$-function components in the basis dictated by the exchange field. Therefore, applying the rotation $\exp \left(i \theta \sigma_{y} / 2\right)$ to $\tilde{\mathbf{h}}, \gamma$, and $\overline{\mathbf{A}}$ yields

$$
\begin{aligned}
f_{0}^{\prime \prime}= & \left(2 \omega_{n}+4 \alpha^{2}\right) f_{0}-4 i \alpha \cos (\theta) f_{1}^{\prime}-4 i \alpha \sin (\theta) f_{3}^{\prime}, \\
f_{1}^{\prime \prime}= & {\left[2 \omega_{n}+4 \alpha^{2} \cos ^{2}(\theta)\right] f_{1}-2 h f_{2}-4 i \alpha \cos (\theta) f_{0}^{\prime} } \\
& +2 \alpha^{2} \sin (2 \theta) f_{3},
\end{aligned}
$$

$f_{2}^{\prime \prime}=2 h f_{1}+2 \omega_{n} f_{2}$,

$f_{3}^{\prime \prime}=\left[2 \omega_{n}+4 \alpha^{2} \sin ^{2}(\theta)\right] f_{3}+2 \alpha^{2} \sin (2 \theta) f_{1}-4 i \alpha \sin (\theta) f_{0}^{\prime}$.

The corresponding equations for $\tilde{f}_{j}$ are obtained after replacing $h \leftrightarrow-h$ and $\alpha \leftrightarrow-\alpha$.

For $h \gg \omega_{n}, \alpha$, it is now straightforward to identify $f_{1}$ and $f_{2}$ as the short-range components, decaying within the length $\xi_{m}=1 / \sqrt{2 h}$ from the interfaces. These two components are the $m=0$ triplet and the singlet component of the pairing amplitude, respectively. These components can thus be solved separately in a straightforward manner, but the resulting analytic expressions are too lengthy to be printed here.

The short-range components generate boundary conditions for the long-range components $f_{0}$ and $f_{3}$ that decay within the length $\ell_{0}=1 / \sqrt{2 \omega_{n}+4 \alpha^{2}}, \ell_{3}=1 / \sqrt{2 \omega_{n}+4 \alpha^{2} \sin (\theta)} \gg \xi_{m}$. First, disregarding the coupling term between $f_{0}$ and $f_{3}$, we get an analytic solution,

$f_{0}=A_{0} \sinh \left[\frac{z}{\ell_{0}}\right]+4 i \alpha \cos (\theta) \int_{0}^{z} \ell_{0} \sinh \left[\frac{z-x}{\ell_{0}}\right] f_{1}^{\prime}(x) d x$,

$f_{3}=A_{3} \sinh \left[\frac{z}{\ell_{3}}\right]+2 \alpha^{2} \sin (2 \theta) \int_{0}^{z} \ell_{3} \sinh \left[\frac{z-x}{\ell_{3}}\right] f_{1}(x) d x$

This solution takes into account the boundary condition $f_{0}(0)=f_{3}(0)=0$. The prefactors $A_{0,3}$ would be obtained from the other boundary condition $f_{0}(1)=f_{3}(0)=1$. However, we concentrate only on the vicinity of the interface at $z=0$ and disregard these terms. Note that both components vanish for $\theta=\pi / 2$, when the exchange field is collinear with the spin-orbit field, whereas $f_{3}=0$ for $\theta=0$.

For $\ell_{0,3} \gg z \gg \xi_{m}$, we may expand and get

$$
\begin{aligned}
& f_{0}(z) \approx 4 i \alpha \cos (\theta) \int_{0}^{z} f_{1}(x) d x, \\
& f_{3}^{\prime}(z) \approx 2 \alpha^{2} \sin (2 \theta) \int_{0}^{z} f_{1}(x) d x .
\end{aligned}
$$

The previous expression requires also one partial integration and using the fact that $f_{1}(0)=0$. The equation is written for $f_{3}^{\prime}(z)$ because only the derivative tends to a constant in this interval. Performing the integral yields $\int_{0}^{z} f_{1}(x) \approx$ $c \exp (i \phi / 2) /(2 \sqrt{h})$, saturating for $z \gg \xi_{m}$. Repeating a similar procedure on the other end, $z=1$ (in reduced units) yields finally the full boundary conditions for the long-range components

$$
\begin{gathered}
f_{0}(0)=2 i \alpha \cos (\theta) c e^{i \phi / 2} / \sqrt{h}, \\
f_{3}^{\prime}(0)=\alpha^{2} \sin (2 \theta) c e^{i \phi / 2} / \sqrt{h}, \\
f_{0}(1)=2 i \alpha \cos (\theta) c e^{-i \phi / 2} / \sqrt{h}, \\
f_{3}^{\prime}(1)=-\alpha^{2} \sin (2 \theta) c e^{-i \phi / 2} / \sqrt{h},
\end{gathered}
$$

where now $z=0,1$ means the position $\sim \xi_{m}$ away from the contacts. The boundary conditions for $\tilde{f}_{0,3}$ are obtained by changing the sign of the derivatives, $\alpha \leftrightarrow-\alpha, \phi \leftrightarrow-\phi$. The remaining equations for the long-range components can be 
written for the two-component vector $\vec{f}=\left(\begin{array}{ll}f_{0} & f_{3}\end{array}\right)^{T}$ as $\vec{f}^{\prime \prime}=\left[\omega_{n}+4 \alpha^{2}\right] \vec{f}-4 \alpha^{2} \cos ^{2}(\theta) \sigma_{\downarrow} \vec{f}-4 i \alpha \sin (\theta) \sigma_{x} \vec{f}^{\prime}$.

We get rid of the second off-diagonal term by defining $\vec{h}=U(z) \vec{f}=\exp \left[-2 i \alpha \sin (\theta) \sigma_{x} z\right] \vec{f}$. This satisfies

$$
\vec{h}^{\prime \prime}=\left[\omega_{n}+4 \alpha^{2} \cos ^{2}(\theta)\right] \vec{h}-4 \alpha^{2} \cos ^{2}(\theta) U(z) \sigma_{\downarrow} U^{\dagger}(z) \vec{h}
$$

with $\quad h_{0}(0)=f_{0}(0), \quad h_{3}^{\prime}(0)=f_{3}^{\prime}(0), \quad\left[\exp \left(2 i \alpha \sin (\theta) \sigma_{x}\right)\right.$ $\vec{h}(1)]_{0}=f_{0}(1), \quad\left[\exp \left(2 i \alpha \sin (\theta) \sigma_{x}\right) \vec{h}^{\prime}(1)\right]_{3}=f_{3}^{\prime}(1)$. Besides Eq. (B18) we can hence transform the coupling terms to the boundary conditions. As the general solution to Eq. (B18) is overly complicated, we disregard the last term in Eq. (B18). In this case, solving (B18) is straightforward. The full solution for $\vec{h}(z)$ is lengthy, but the spectral supercurrent is given by $j_{s}=8 i \alpha^{2} \cos ^{2}(\theta) c^{2} \sin (\phi) I(\alpha)$ with

$$
I(\alpha)=\frac{e^{\sqrt{2} \sqrt{\widetilde{\omega}_{n}}}\left(\sqrt{2}\left(e^{2 \sqrt{2} \sqrt{\tilde{\omega}_{n}}}-1\right) \cos (2 \alpha \sin (\theta))\left(2 \tilde{\omega}_{n}-\alpha^{2} \sin ^{2}(\theta)\right)-4 \alpha \sin (\theta)\left(e^{2 \sqrt{2} \sqrt{\widetilde{\omega}_{n}}}+1\right) \sqrt{\widetilde{\omega}_{n}} \sin (2 \alpha \sin (\theta))\right)}{h \sqrt{\widetilde{\omega}_{n}}\left(-2 e^{2 \sqrt{2} \sqrt{\tilde{\omega}_{n}}} \cos (4 \alpha \sin (\theta))+e^{4 \sqrt{2} \sqrt{\tilde{\omega}_{n}}}+1\right)},
$$

where $\tilde{\omega}_{n}=\omega_{n}+2 \alpha^{2} \cos ^{2}(\theta)$. In particular, for $\theta=0$, where the above approximation of neglecting the last term in Eq. (B18) is not relevant, we get Eq. (15).

For $\theta$ between 0 and $\pi / 2$, the two long-range components mix and produce a supercurrent that can change sign for a fixed phase as the direction of the magnetic field is tuned. Such a sign change is described by the terms of the form $\cos (2 \alpha \sin (\theta))$ that result from the above transformation between $\vec{f}$ and $\vec{h}$. They show that the sign change takes place whenever $\alpha \gtrsim 1$. However, due to the approximation of neglecting the last term in Eq. (B18), this result does not match very well the exact solution at arbitrary range of parameters. Therefore, it is better to use the rather straightforward solution of the full linearized equations for finding the supercurrent in this case.
[1] V. V. Ryazanov, V. A. Oboznov, A. Y. Rusanov, A. V. Veretennikov, A. A. Golubov, and J. Aarts, Phys. Rev. Lett. 86, 2427 (2001).

[2] T. Kontos, M. Aprili, J. Lesueur, and X. Grison, Phys. Rev. Lett. 86, 304 (2001).

[3] F. S. Bergeret, A. F. Volkov, and K. B. Efetov, Phys. Rev. Lett. 86, 4096 (2001).

[4] T. S. Khaire, M. A. Khasawneh, W. P. Pratt, and N. O. Birge, Phys. Rev. Lett. 104, 137002 (2010).

[5] J. W. A. Robinson, J. D. S. Witt, and M. G. Blamire, Science 329, 59 (2010).

[6] T. T. Heikkilä, F. K. Wilhelm, and G. Schön, Europhys. Lett. 51, 434 (2000).

[7] S.-K. Yip, Phys. Rev. B 62, R6127(R) (2000).

[8] M. S. Crosser, J. Huang, F. Pierre, P. Virtanen, T. T. Heikkilä, F. K. Wilhelm, and N. O. Birge, Phys. Rev. B 77, 014528 (2008).

[9] J. C. Cuevas and F. S. Bergeret, Phys. Rev. Lett. 99, 217002 (2007).

[10] V. Mourik, K. Zuo, S. M. Frolov, S. R. Plissard, E. P. A. M. Bakkers, and L. P. Kouwenhoven, Science 336, 1003 (2012).

[11] A. Das, Y. Ronen, Y. Most, Y. Oreg, M. Heiblum, and H. Shtrikman, Nat. Phys. 8, 887 (2012).

[12] H. O. H. Churchill, V. Fatemi, K. Grove-Rasmussen, M. T. Deng, P. Caroff, H. Q. Xu, and C. M. Marcus, Phys. Rev. B 87, 241401 (2013).

[13] J. D. Sau, R. M. Lutchyn, S. Tewari, and S. Das Sarma, Phys. Rev. Lett. 104, 040502 (2010).

[14] Y. Oreg, G. Refael, and F. von Oppen, Phys. Rev. Lett. 105, 177002 (2010).

[15] K. Sun and N. Shah, Phys. Rev. B 91, 144508 (2015).

[16] Francesco Giazotto, Attila Geresdi, and Charles Marcus (private communications).
[17] J. Paajaste, M. Amado, S. Roddaro, F. S. Bergeret, D. Ercolani, L. Sorba, and F. Giazotto, Nano Lett. 15, 1803 (2015).

[18] F. Giazotto, P. Spathis, S. Roddaro, S. Biswas, F. Taddei, M. Governale, and L. Sorba, Nat. Phys. 7, 857 (2011).

[19] P. Spathis, S. Biswas, S. Roddaro, L. Sorba, F. Giazotto, and F. Beltram, Nanotechnology 22, 105201 (2011).

[20] S. Roddaro, A. Pescaglini, D. Ercolani, L. Sorba, F. Giazotto, and F. Beltram, Nano Res. 4, 259 (2011).

[21] I. van Weperen, S. R. Plissard, E. P. A. M. Bakkers, S. M. Frolov, and L. P. Kouwenhoven, Nano Lett. 13, 387 (2013).

[22] O. Gül, D. J. van Woerkom, I. van Weperen, D. Car, S. R. Plissard, E. P. A. M. Bakkers, and L. P. Kouwenhoven, Nanotechnology 26, 215202 (2015).

[23] I. van Weperen, B. Tarasinski, D. Eeltink, V. S. Pribiag, S. R. Plissard, E. P. A. M. Bakkers, L. P. Kouwenhoven, and M. Wimmer, Phys. Rev. B 91, 201413 (2015).

[24] P. Dubos, H. Courtois, B. Pannetier, F. K. Wilhelm, A. D. Zaikin, and G. Schön, Phys. Rev. B 63, 064502 (2001).

[25] H. le Sueur, P. Joyez, H. Pothier, C. Urbina, and D. Esteve, Phys. Rev. Lett. 100, 197002 (2008).

[26] D. Bagrets and A. Altland, Phys. Rev. Lett. 109, 227005 (2012).

[27] D. I. Pikulin, J. P. Dahlhaus, M. Wimmer, H. Schomerus, and C. W. J. Beenakker, New J. Phys. 14, 125011 (2012).

[28] P. Neven, D. Bagrets, and A. Altland, New J. Phys. 15, 055019 (2013).

[29] F. S. Bergeret and I. V. Tokatly, Phys. Rev. Lett. 110, 117003 (2013).

[30] F. S. Bergeret and I. V. Tokatly, Phys. Rev. B 89, 134517 (2014).

[31] C. Gorini, P. Schwab, R. Raimondi, and A. L. Shelankov, Phys. Rev. B 82, 195316 (2010).

[32] K. D. Usadel, Phys. Rev. Lett. 25, 507 (1970).

[33] W. Belzig, F. K. Wilhelm, C. Bruder, G. Schön, and A. D. Zaikin, Superlattices Microstruct. 25, 1251 (1999). 
[34] Note that our representation differs by that used, for example, in Refs. $[33,39]$. The two representations are linked via a unitary transformation $\hat{U}=\left(1-i \hat{\tau}_{3} \bar{\sigma}_{2}\right)\left(1+i \bar{\sigma}_{2}\right) / 2$ to the Green's functions and the components of the Usadel equation.

[35] A. Abrikosov and L. Gor'kov, Sov. Phys. JETP 15, 752 (1962).

[36] M. Eschrig, Phys. Rev. B 61, 9061 (2000).

[37] T. Matsubara, Prog. Theor. Phys. 14, 351 (1955).

[38] J. Cayao, E. Prada, P. San-Jose, and R. Aguado, Phys. Rev. B 91, 024514 (2015).

[39] J. Rammer and H. Smith, Rev. Mod. Phys. 58, 323 (1986).

[40] T. T. Heikkilä, J. Särkkä, and F. K. Wilhelm, Phys. Rev. B 66, 184513 (2002).

[41] J. Arjoranta, Master's thesis, University of Helsinki, 2014 .

[42] S. H. Jacobsen and J. Linder, Phys. Rev. B 92, 024501 (2015).

[43] S. H. Jacobsen, J. A. Ouassou, and J. Linder, Phys. Rev. B 92, 024510 (2015).

[44] A. I. Buzdin, L. N. Bulaevskii, and S. V. Panyukov, JETP Lett. 35, 178 (1982).
[45] I. V. Krive, L. Y. Gorelik, R. I. Shekhter, and M. Jonson, Low Temp. Phys. 30, 398 (2004).

[46] F. S. Bergeret and I. V. Tokatly, Europhys. Lett. 110, 57005 (2015).

[47] F. S. Bergeret, A. F. Volkov, and K. B. Efetov, Phys. Rev. B 64, 134506 (2001).

[48] F. Zhou, P. Charlat, B. Spivak, and B. Pannetier, J. Low Temp. Phys. 110, 841 (1998).

[49] See https://github.com/wompo/Usadel-for-nanowires for more examples on the form of the local density of states.

[50] Y. Tanaka and A. A. Golubov, Phys. Rev. Lett. 98, 037003 (2007).

[51] A. Konstandin, J. Kopu, and M. Eschrig, Phys. Rev. B 72, 140501 (2005).

[52] M. Alidoust, G. Rashedi, J. Linder, and A. Sudbø, Phys. Rev. B 82, 014532 (2010).

[53] M. Alidoust, K. Halterman, and O. T. Valls, Phys. Rev. B 92, 014508 (2015).

[54] A. Shelankov, J. Low Temp. Phys. 60, 29 (1985). 